\title{
Network Formation Among Selfish Energy-Constrained Wireless Devices
}

\author{
Hithesh Nama, Narayan Mandayam, Roy Yates \\ WINLAB, Rutgers University \\ 671 Route 1 South, North Brunswick, NJ 08902 \\ Email: $\{$ hithesh, narayan, ryates\}@ winlab.rutgers.edu
}

\begin{abstract}
We study the formation of ad-hoc networks among selfish energy-constrained wireless devices that are primarily interested in being connected with other devices. We use a non-cooperative bilateral connection game (BCG) framework to study network formation. For a BCG in which devices choose their individual strategies to remain connected by minimizing only their direct transmission power costs, we show that the price-of-anarchy is unbounded in the network size. We propose a BCG with an alternate cost structure in which each device additionally pays the transmission power costs incurred by other devices for its own traffic. We show that a unique network structure emerges in this game that is stable as well as socially efficient. We then study the achievable throughput for random point-to-point traffic in this stable energy-efficient network. When the nodes of a network are located in a bounded planar region the distribution of pointto-point flows through the nodes exhibits a scale-free behavior.
\end{abstract}

\section{INTRODUCTION}

\section{A. Motivation}

In recent years, several researchers have started studies to better understand the reasons behind the enormous growth in the size of the Internet. At a certain abstraction, the Internet is but a network of selfish autonomous subnetworks. Hosts within a sub-network would like to be able to connect to hosts in another sub-network. However, connectivity entails a cost. Sub-networks are typically owned by business entities seeking to maximize their revenue from the hosts they serve, while keeping their connectivity cost at a minimum. Driven by market forces and with a strong underlying protocol structure the Internet has come to become one of the largest distributed systems ever to be built.

Networks that are created and sustained through uncoordinated interactions among multiple selfish entities, in a manner similar to the Internet, appear in a wide variety of situations like social networks of individuals, trade networks, political alliances, research collaborations among firms, the world wide web, and peer-to-peer systems for file sharing. However, in the context of wireless networks, which are the focus of our work, a great majority of those that are in operation today are carefully planned centrally coordinated networks. Inspired by the Internet and the various other networks mentioned above, in this work, we explore the possibility of network formation among the myriad portable wireless devices - like hand-held mobile phones, Bluetooth-enabled PDAs, WiFi laptops, etc., - in an uncoordinated manner without any explicit centralized coordinating mechanism.

We focus specifically on the formation of a connected network among a bunch of wireless devices i.e., a network in which any two devices or nodes are connected either directly or indirectly through multiple hops over other nodes. Consider a scenario in which each node has a specific destination that it wishes to communicate with but the location of the destination changes in a quasistatic manner. In another scenario, a node might wish to communicate with any one node from among several nodes in a certain geographical area. Situations such as these in which there is an uncertainty either in the location of the destination or the identity of the destination require that all the nodes form a connected network among themselves.

An important feature of portable wireless devices is that they are battery powered and therefore energy is a premium. It is thus natural that each of these devices, though interested in forming a network with the others, would like to do so by expending as little power as possible on its part. While short links with low transmission power costs are favored to conserve power, they may not lead to a connected network. The conflict between the need for connectivity and the selfish objective of conserving energy motivates us to question whether selfish energyconstrained wireless devices do indeed form connected networks. Further, if the devices form a connected network, we wish to study how it compares with the network formed in a centrally coordinated manner in terms of the total energy dissipation or the energy efficiency.

\section{B. Prior Work}

Game-theoretic models have been a natural choice to study networks that emerge from uncoordinated actions among selfish agents or players. A simple but insightful model consists of a single stage link connection game in which players simultaneously announce the list of other players that they wish to be connected to. Based on all the announcements, links are formed between players according to a linking rule and thus a network is formed among the players. Each player incurs a cost in this network, which is a function of all the announcements, and the player's own 
announcement seeks to minimize this cost. The social cost of a network is defined as the sum of costs incurred by the individual players and socially optimal networks are those that have the least social cost.

In the unilateral connection game of [1], the linking rule dictates that a link is formed between two players if either one of the players announce an interest to form the link and that it can then be used by both the players. Such a game is used to model the creation of Internet-like networks. Each player's cost function is a weighted sum of two components: the total cost of links announced by this player and the sum of distances (hop counts) from this player to all others. Networks that are Nash equilibria of the unilateral connection game are the stable networks in this model and they are not necessarily socially efficient. There is thus a conflict between stability and social efficiency. In order to study the loss in efficiency due to uncoordinated network formation, the authors evaluate the price-of-anarchy [2], which is the ratio of the social costs of the worst-case Nash equilibrium network and the socially efficient network.

Another linking rule in which a link is formed between two players only if both of them announce an interest to connect is proposed in [3]. [4] uses this model, also called a bilateral connection game (BCG), together with the cost function of [1] to show that there is once again a conflict between stability and social efficiency and that in fact the price-of-anarchy of the bilateral model is worse than that for the unilateral model. The notion of stability (Nash equilibria) used in unilateral connection games needs to be refined in the context of bilateral connection games in order to sift out useful networks from among the multitude of Nash equilibrium networks. A refinement of Nash equilibrium called pairwise-Nash equilibrium is defined in [5] where players are also allowed to deviate by pairs unlike only unilateral deviations in a Nash equilibrium. We discuss these concepts in detail in Section II-B.

The conflict between stability and social efficiency seen in models of the Internet ( [1], [4]) has also been observed in a wide variety of other network models that include social networks, trade networks, political alliances, research collaborations among firms, the world wide web, and peerto-peer systems for file sharing. [6] provides an excellent survey of several such models and explores the inherent tension between stability and social efficiency in networks formed from uncoordinated actions of selfish players. In contrast to uncoordinated network formation studied in this work, [7] studies formation of coalitions in cooperative wireless networks using game theoretic and information theoretic models.

\section{Overview}

In this work, we use a non-cooperative BCG framework to model network formation among energy-constrained wireless devices deployed over a bounded region in the two dimensional plane. Each device or player is interested in being connected to every other player but with the least power. Therefore, unlike the constant link costs in [1] and [4], we associate a power cost with a link, which is the minimum power required for successfully communicating on this link. We assume a large scale path-loss model and therefore the power cost is proportional to $d^{\alpha}$, where $d$ is the link length and $\alpha$ is the path-loss exponent. Communication between two players that are not connected directly takes place along the least-cost route. As mentioned earlier, our focus in this work is on forming connected networks and therefore we assume an infinite route cost if two players are not connected to each other in the network. The more general case where a node only wishes to connect to a subset of all other nodes is not considered in this work and is currently under investigation. We however emphasize that studying the formation of connected networks is important for practical situations as stated before and also yields valuable insights.

Each player in the BCG chooses the list of other players that it wishes to directly connect to based on the criteria of minimizing its own cost. We first consider the case where the cost incurred by a player is the sum of transmission power costs over the direct links. For a BCG in which devices choose their individual strategies to remain connected by minimizing such a natural cost function, we show that the price-of-anarchy is unbounded in the network size. Therefore, stable networks comprising a large number of nodes with such a natural cost function can have very high social costs i.e., they can be highly energy inefficient. We then propose an alternate cost function in which each device additionally pays the transmission power costs incurred by other devices for its own traffic. Such a payment can be implemented using a virtual energy currency on the lines of the nuglets-based Packet Purse Model of [8]. An important feature of a BCG with the proposed cost function is that a unique network structure emerges in this game that is stable as well as socially efficient i.e., the network formed through uncoordinated selfish actions is the same as the energy efficient network formed using a central coordinator.

The energy constrained nature of wireless devices has motivated us to study network formation while each node tries to minimize its power costs. Since we did not explicitly consider the effects of interference due to simultaneous wireless transmissions, for the stable energy-efficient network that results from our BCG, we study the spectral efficiency achievable for routing multiple point-to-point flows to random destinations. Recently, [9], [10], [11] have studied the capacity of a wireless network consisting of $n$ nodes inside a bounded region on a plane with a flow from each node to a random destination. It is shown that the capacity of each flow decreases as $1 / \sqrt{n \log n}$ as the number of nodes increases. For the stable energy-efficient network obtained in our model, based on analysis and empirical observations, we conjecture that the capacity of individual flows for random point to point traffic is order optimal i.e., the throughput achieved by each flow is $\Omega(1 / \sqrt{n \log n})$. 
When we consider random point to point flows among nodes deployed in a square region, nodes at the center of the region are more likely to route a higher number of flows than those that are far away from the center. Interestingly, we observe a "scale-free" or "power-law" distribution for the number of flows that pass through any node in the stable energy-efficient network. The degree distribution of large networks like the Internet and several social networks has been empirically observed to approximate a scalefree distribution and has implications in system design. [12], [13], and [14] propose network formation models that generate networks in which, among other features, the node degree distribution resembles a scale free distribution.

In the next section we present the BCG model and define notions of stability and efficiency. In Section III we discuss stability and efficiency of networks resulting from our BCG model. The throughput that can be achieved by each flow for multiple random point-to-point flows over the stable energy-efficient network is studied analytically in Section IV.

\section{SYSTEM MODEL}

\section{A. Bilateral Connection Game}

Consider a link connection game in a set $N=\{1, \ldots, n\}$ of wireless devices or players that are located on a twodimensional unit square. The strategy space of the $i^{\text {th }}$ player in this game is the set $\mathcal{S}_{i}=\{0,1\}^{n-1}$ of size $2^{n-1}$. A strategy $\mathbf{s}_{i} \in \mathcal{S}_{i}$ is a vector of length $n-1$ with $\mathbf{s}_{i j}=1, j \neq i$ indicating player $i$ 's consent to connect to player $j$. We consider a single stage game in which all players announce their strategies simultaneously. Let $\mathbf{s}=\left(\mathbf{s}_{1}, \ldots, \mathbf{s}_{n}\right) \in \mathcal{S}_{1} \times \ldots \times \mathcal{S}_{n}$ denote the strategy profile announced by all players and $\mathbf{s}_{-i}$ denote the strategy profile of all players excluding the $i^{\text {th }}$ player. Given s, we form the undirected graph $G(\mathbf{s})=(N, B(\mathbf{s}))$ with vertex set $N$ comprising of players and edge set $B(\mathbf{s})$ consisting of communication links between players. Link formation is based on mutual consent i.e., $B(\mathbf{s})=\{(i, j) \mid i \neq$ $\left.j, \mathbf{s}_{i j} \mathbf{s}_{j i}=1\right\}$ and hence the game is called a Bilateral Connection Game (BCG).

Let $c_{i j}$ denote the power cost of link $(i, j) \in B(\mathbf{s})$, which is the minimum transmit power required for successfully communicating on this link. We assume that transmissions are power controlled to ensure a certain minimum signal-tonoise ratio (SNR) at the receiver that guarantees successful reception. Therefore, $c_{i j}=c_{0} d_{i j}^{\alpha}$ where $c_{0}$ is a constant, $d_{i j}$ is the distance between nodes $i$ and $j$, and $\alpha \geq 2$ is the path loss exponent. We define $c_{i i}=0$. The power cost of a path in $G(\mathbf{s})$ is the sum of power costs of edges along this path. Let $\hat{c}_{i j}(\mathbf{s})$ denote the power cost of the least-cost path between nodes $i$ and $j$ in $G(\mathbf{s})$. We assume $\hat{c}_{i j}(\mathbf{s})=\infty$ if nodes $i$ and $j$ are not connected in $G(\mathbf{s})$. We consider two different node cost functions in our model. The first cost function that involves power costs of direct links alone is what is observed naturally and is defined as

$$
C_{i}^{(1)}(\mathbf{s})= \begin{cases}\sum_{j} c_{i j} s_{i j} & \text { if } G(\mathbf{s}) \text { is connected } \\ \infty & \text { otherwise }\end{cases}
$$

We show in the sequel that networks formed in a BCG with this naturally observed cost function are asymptotically inefficient. We therefore propose an alternative node cost function that in addition to the power costs over direct links also includes the transmission power costs incurred by other devices for the node's own traffic

$$
C_{i}^{(2)}(\mathbf{s})=\sum_{j} c_{i j} s_{i j}+\sum_{j \mid(i, j) \notin B(\mathbf{s})} \hat{c}_{i j}(\mathbf{s}) .
$$

We show that networks formed in a BCG with this second cost function are both stable as well as energy efficient. Players are selfish in that they choose their individual strategies so as to minimize their costs. Given a particular node cost function, we define the social cost of a graph as follows:

Definition 2.1: The social cost of a graph is defined as the sum of costs of individual players in that graph. The graph with the least social cost is said to be socially efficient.

A BCG with cost function as in (1) might seem more 'natural' because in practice each node in the network expends power only on its direct links and on its own link announcements. However, as shown in the sequel a BCG with such a cost function can result in networks that are increasingly inefficient compared to the socially efficient network as the size of the network increases. We therefore propose a BCG with cost function as in (2) in which each node also bears the cost of least-cost paths from itself to other nodes in the network that are not directly connected to it. We show that networks resulting from such a BCG have interesting stability and efficiency characteristics that could be of interest in network formation among selfish wireless devices.

\section{B. Pairwise Stability and Pairwise-Nash Equilibrium}

We are interested in studying the stability of graphs that result from the BCG and therefore Nash graphs, which result from strategy profiles that are Nash equilibria of the BCG are of immediate interest. In the sequel, we use $C_{i}(\mathbf{s})$ without a superscript to denote either $C_{i}^{(1)}(\mathbf{s})$ or $C_{i}^{(2)}(\mathbf{s})$ and when a specific cost function is implied the superscript will be indicated explicitly.

Definition 2.2: A graph $G(\mathbf{s})$ is a Nash graph if the strategy profile $\mathbf{s}$ is a Nash equilibrium of the BCG i.e., $C_{i}(\mathbf{s})=C_{i}\left(\mathbf{s}_{i}, \mathbf{s}_{-i}\right) \leq C_{i}\left(\mathbf{s}_{i}^{\prime}, \mathbf{s}_{-i}\right)$ for all players $i$.

Two important properties of Nash graphs follow immediately based on the structure of the game described above.

- In a Nash graph, the strategy of a player does not involve unilateral link announcements. This is because nodes incur a cost from unilateral link announcements and by deviating from a strategy containing such 
announcements, nodes necessarily reduce their overall cost (see (1) and (2)).

- Unilateral strategy deviations from a Nash graph can only result in link deletions. This is because link formation requires mutual consent but link deletion does not.

Note that a multitude of Nash graphs exist for the BCG with cost functions as given by (1) and (2). For instance, the strategy profile with $s_{i j}=0, i \in N, j \in N$, which results in the empty graph i.e., $B(\mathbf{s})=\phi$ is a Nash graph. Similarly, any strategy profile that results in a tree graph and that does not involve unilateral announcements (i.e., $s_{i j}=1$ but $s_{j i}=0$ for some $i$ and $j$ ) is also a Nash graph. Therefore, in order to study graphs that are more interesting both from a theoretical and practical perspective, we now consider a refinement of the definition of Nash graph.

For a strategy profile $\mathbf{s}$, let $\mathbf{s}+(i, j)$ denote a similar strategy profile that also includes link $(i, j)$ i.e., $B(\mathbf{s}+$ $(i, j))=B(\mathbf{s}) \cup\{(i, j)\}$. Similarly, let $\mathbf{s}^{\prime}=\mathbf{s}-(i, j)$ denote a deviated strategy profile in which $\mathbf{s}_{i j}^{\prime}=0$ and therefore $(i, j) \notin B(\mathbf{s}-(i, j))$. We define a refinement of a Nash graph called pairwise-Nash graph [5] such that every mutually beneficial link is an edge in this graph.

Definition 2.3: A graph $G(\mathbf{s})$ is pairwise-Nash if $G(\mathbf{s})$ is a Nash graph and for all $(i, j) \notin B(\mathbf{s})$, if $C_{i}(\mathbf{s}+(i, j)) \leq$ $C_{i}(\mathbf{s})$ then $C_{j}(\mathbf{s}+(i, j))>C_{j}(\mathbf{s})$.

An intuitive interpretation of pairwise-Nash graphs is that if players are allowed to coordinate bilaterally then no mutually beneficial link is left aside. Pairwise-Nash graphs are the Nash equilibrium outcomes of the BCG that fulfill this added coordinated move requirement. Let $\pi\left(C_{i}(\mathbf{s})\right)$ denote the set of all pairwise-Nash networks for a BCG with cost function $C_{i}(\mathbf{s})$.

We now define pairwise-stability which is a simpler notion of stability involving only one-link deviations unlike multi-link deviations in the case of Nash or PairwiseNash graphs. Given any graph, pairwise-stability is an easier condition to verify than the Nash or pairwise-Nash conditions and will simplify our subsequent analysis.

Definition 2.4: A graph $G(\mathbf{s})$ is pairwise-stable if $\mathbf{s}$ does not contain any unilateral announcements and if for all $(i, j) \in B(\mathbf{s}), C_{i}(\mathbf{s}-(i, j)) \geq C_{i}(\mathbf{s})$, while for all $(i, j) \notin$ $B(\mathbf{s})$, if $C_{i}(\mathbf{s}+(i, j)) \leq C_{i}(\mathbf{s})$ then $C_{j}(\mathbf{s}+(i, j))>C_{j}(\mathbf{s})$. Thus, every edge in a pairwise-stable graph $G(\mathbf{s})$ is a mutually beneficial link and every mutually beneficial link is an edge of $G(\mathbf{s})$.

\section{Stability AND EFFiciency OF Networks IN THE $\mathrm{BCG}$}

Theorem 3.1: For the BCG with cost function $C_{i}^{(1)}(\mathbf{s})$ given in (1), a graph $G(\mathbf{s})$ is pairwise-stable if and only if it is pairwise-Nash.

Proof: It is easy to verify from the definitions that every pairwise-Nash graph is pairwise-stable irrespective of the cost function. However the converse is not true in general for every cost function. In order to show the converse, we only need to consider the case of deletion of links $(i, j) \in B(\mathbf{s})$ since constraints on links $(i, j) \notin$ $B(\mathbf{s})$ are the same for pairwise stability and pairwise-Nash. In particular, we need to verify that for the BCG with cost function $C_{i}^{(1)}(\mathbf{s})$, a node in a pairwise stable graph $G(\mathbf{s})$ does not benefit from deleting multiple links i.e. the marginal cost of deleting multiple links is non-negative.

Consider a pairwise stable graph $G(\mathbf{s})$. If $G(\mathbf{s})$ is not connected, then $C_{i}(\mathbf{s})=\infty, i \in N$ and it remains so whether a node deletes any single direct link or multiple direct links. If $G(\mathbf{s})$ is connected, then s does not contain any unilateral link announcements and hence $C_{i}^{(1)}(\mathbf{s})=$ $\sum_{j \mid(i, j) \in B(\mathbf{s})} c_{i j}(\mathbf{s})<\infty$. The marginal cost of deleting multiple links is either infinite (when the resulting network is not connected) or equal to the sum of power costs of the deleted links, which is non-negative. Thus no node in a pairwise stable graph benefits from deleting either one or more of its links. Every pairwise stable graph is therefore equivalent to a pairwise-Nash graph for the BCG with cost function $C_{i}^{(1)}(\mathbf{s})$.

Theorem 3.2: For the BCG with cost function $C_{i}^{(1)}(\mathbf{s})$ as in (1), a graph $G(\mathbf{s})$ is pairwise-Nash if and only if it is a tree and $\mathbf{s}$ does not contain any unilateral link announcements.

Proof: If a pairwise-stable graph $G(\mathbf{s})$ is connected but is not a tree, then the cost of node $i$ is $C_{i}^{(1)}(\mathbf{s})=$ $\sum_{j \mid(i, j) \in B(\mathbf{s})} c_{i j}$ which can be reduced by deleting existing direct links so long as the graph is still connected. Thus existing links in this graph are not beneficial and hence the graph is not pairwise-stable from Definition 2.4 and consequently not pairwise-Nash from Theorem 3.1,

For the converse, if $\mathbf{s}$ does not contain any unilateral link announcements and the graph $G(\mathbf{s})$ is a tree, then from $(1), C_{i}^{(1)}(\mathbf{s})=\sum_{j \mid(i, j) \in B(\mathbf{s})} c_{i j}$. Deleting an existing link $(i, j) \in B(\mathbf{s})$ will disconnect the network and result in an infinite cost for the end nodes $i$ and $j$. Every existing link is thus mutually beneficial. Adding a link $(i, j) \notin B(\mathbf{s})$ will only increase the cost function of both end nodes and thus no mutually beneficial link is absent from $G(\mathbf{s})$. Therefore $G(\mathbf{s})$ is pairwise-stable and consequently pairwise-Nash from Theorem 3.1.

As mentioned in Section I, the price-of-anarchy [2] is a widely used metric to study the loss in efficiency due to uncoordinated network formation compared to centralized network formation. We now give a formal definition for the price-of-anarchy in a BCG.

Definition 3.3: For a BCG with cost function $C_{i}(\mathbf{s})$, the price-of-anarchy $\eta\left(C_{i}(\mathbf{s})\right)$ is defined as the ratio of the social costs of the worst-case pairwise-Nash network and the socially efficient network i.e.,

$$
\eta\left(C_{i}(\mathbf{s})\right)=\frac{\max _{G(\mathbf{s}) \in \pi\left(C_{i}(\mathbf{s})\right)} \sum_{i \in N} C_{i}(\mathbf{s})}{\min _{G(\mathbf{s})} \sum_{i \in N} C_{i}(\mathbf{s})} .
$$

We now describe a model for studying asymptotic properties of random networks. Indexed by the number of nodes $n$, we construct a sequence of random networks using the 
BCG framework described above. Each node in a sequence of networks is deployed independently (of other nodes) and randomly with a uniform distribution on the unit square. Consider a sequence of events $E_{1}, \ldots, E_{n}$ with $E_{n}$ corresponding to the event in the network with $n$ nodes. We say that these events occur almost surely if their complementary sequence of events do not occur infinitely often with probability one i.e., $P\left(\left\{E_{n}^{C}\right.\right.$ occurs infinitely often $\left.\}\right)=0$. Now for a network of $n$ nodes, we partition the unit square into a grid of $1 / A_{n}$ cells each of area $A_{n}$ as shown in Fig. 1 and indexed as $\mathcal{C}_{1}, \ldots, \mathcal{C}_{1 / A_{n}}$. With an abuse of notation, we use $\mathcal{C}_{i}$ to indicate the $i^{t h}$ cell as well as the number of nodes in that cell.

Lemma 3.4: For $A_{n}=\frac{a \log n}{n}, a>2$, each cell has $\Theta(\log n)$ nodes almost surely.

Proof: Let $Y_{i}$ be a Bernoulli random variable with $Y_{i}=1$ if node $i$ lies in cell $\mathcal{C}_{1}$. We need to show that the sequence of events $E_{n}=\cap_{i=1}^{n}\left\{\mathcal{C}_{i}=\Theta(\log n)\right\}$ occurs almost surely or equivalently their complementary events do not occur infinitely often with probability one.

$$
\begin{aligned}
P\left(\bigcup_{i=1}^{n}\left\{\mathcal{C}_{i}>b \log n\right\}\right) \leq n P\left(\mathcal{C}_{1}>b \log n\right) \\
\quad=n P\left(Y_{1}+\cdots+Y_{n}>b \log n\right) \\
\quad \leq n \frac{E\left[\exp \left(\theta \sum_{j=1}^{n} Y_{j}\right)\right]}{\exp (\theta b \log n)} \\
\quad=n \frac{\left\{E\left[\exp \left(\theta Y_{1}\right)\right]\right\}^{n}}{\exp (\theta b \log n)} \\
\quad=n \frac{\left[e^{\theta} A_{n}+1-A_{n}\right]^{n}}{\exp (\theta b \log n)} \\
\leq n \exp \left(n A_{n}\left(e^{\theta}-1\right)\right) \exp (-\theta b \log n) \\
\quad=n^{1+a\left(e^{\theta}-1\right)-b \theta} \\
=\frac{1}{n^{1+\epsilon}}
\end{aligned}
$$

where we use the union bound in (4a), Chernoff bound in (4c), independence of $\left\{Y_{i}\right\}$ in (4d), and the inequality $1+x \leq e^{x}$ in (4f). Finally, (4h) holds for any $\epsilon>0$ if $b=\left(2+a\left(e^{\theta}-1\right)+\epsilon\right) / \theta$. For a given $a>2$ we can optimize over all $\theta>0$ to find the smallest such value of $b$ for which (4h) holds. Using the Borel-Cantelli lemma, we conclude that each cell has $O(\log n)$ nodes almost surely, since $\sum_{n=1}^{\infty} \frac{1}{n^{1+\epsilon}}<\infty$ for $\epsilon>0$.

Similarly,

$$
\begin{aligned}
P\left(\bigcup_{i=1}^{n}\left\{\mathcal{C}_{i}<b^{\prime} \log n\right\}\right) \leq n P\left(\mathcal{C}_{1}<b^{\prime} \log n\right) \\
\quad=n P\left(Y_{1}+\cdots+Y_{n}<b^{\prime} \log n\right) \\
\leq n \frac{E\left[\exp \left(-\theta^{\prime} \sum_{j=1}^{n} Y_{j}\right)\right]}{\exp \left(-\theta^{\prime} b^{\prime} \log n\right)} \\
=n^{1-a\left(1-e^{-\theta^{\prime}}\right)+b^{\prime} \theta^{\prime}} \\
=\frac{1}{n^{1+\epsilon^{\prime}}}
\end{aligned}
$$

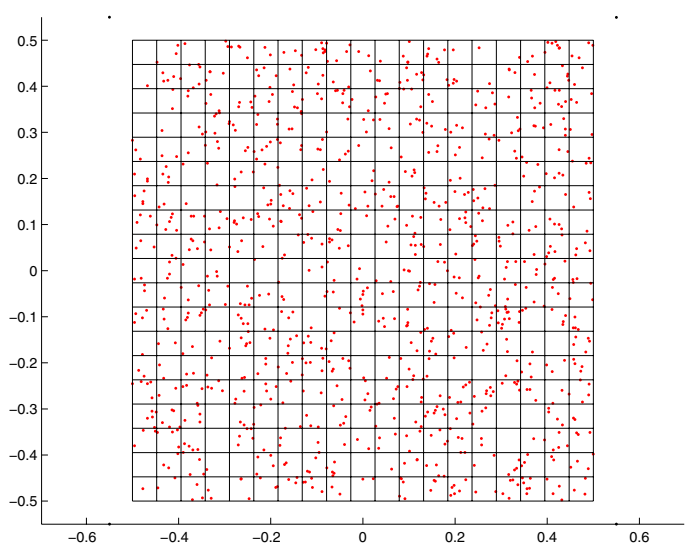

Fig. 1. Partition of the square region into square grids each of area $A_{n}$

where $\theta^{\prime}>0$ and the last equality follows for any $\epsilon^{\prime}>0$ if $b^{\prime}=\left(a\left(1-e^{-\theta^{\prime}}\right)-\left(2+\epsilon^{\prime}\right)\right) / \theta^{\prime}$. For $b^{\prime}>0, a>$ $\left(2+\epsilon^{\prime}\right) /\left(1-e^{-\theta^{\prime}}\right)$ necessarily. We can optimize over all $\theta^{\prime}>0$ to find the largest value of $b^{\prime}$ for which (5e) holds. Using the Borel-Cantelli lemma, we conclude that each cell has $\Omega(\log n)$ nodes almost surely.

Theorem 3.5: For the BCG with cost function $C_{i}^{(1)}(\mathbf{s})$ as in (1), the price-of-anarchy is unbounded almost surely.

Proof: From Definition 3, the price-of-anarchy is lower bounded by the ratio of social costs of any pairwiseNash network and the socially efficient network of the BCG. Consider a partitioning of the unit square into cells of size $A_{n}=\frac{3 \log n}{n}$ where the number of nodes $n$ is sufficiently large so that every cell has $\Theta(\log n)$ nodes almost surely, from Lemma 3.4.

Let $\mathbf{s}_{1}$ denote a strategy profile without any unilateral link announcements such that $G\left(\mathbf{s}_{1}\right)$ is a tree in which each node is connected directly to the node closest to the center of the square. Note that $G\left(\mathbf{s}_{1}\right)$ is a pairwise-Nash graph from Theorem 3.2. The social cost of $G\left(\mathbf{s}_{1}\right)$ can be lower bounded as

$$
C^{(1)}\left(\mathbf{s}_{1}\right)=2 \sum_{(i, j) \in B\left(\mathbf{s}_{1}\right)} c_{i j}>\frac{c_{0} \log n}{2 A_{n}}(1 / 4)^{\alpha}=\frac{c_{0} / 6}{4^{\alpha}} n .
$$

by considering costs of only those nodes that are $1 / 4$ units away from the center of the unit square.

Let $\mathbf{s}_{2}$ denote another strategy profile without any unilateral link announcements such that $G\left(\mathbf{s}_{2}\right)$ is a tree in which no link is longer than $\sqrt{8 A_{n}}$. Such a tree can be constructed based on Lemma 3.4 which guarantees that no cell is empty for the above choice of cell size. The social cost of the socially efficient network can be bounded above as,

$$
\min _{G(\mathbf{s})} \sum_{i \in N} C_{i}^{(1)}(\mathbf{s})<C^{(1)}\left(\mathbf{s}_{2}\right)<c_{0} n(24 \log n / n)^{\alpha / 2} .
$$

Using (6) and (7), the price-of-anarchy (defined in (3)) can be bounded below as

$$
\eta\left(C^{(1)}(\mathbf{s})\right)>c_{1}\left(\frac{n}{\log n}\right)^{\alpha / 2} \rightarrow \infty,
$$


where $c_{1}$ is a positive constant independent of $n$.

Theorem 3.6: For the BCG with cost function $C_{i}^{(2)}(\mathbf{s})$ given in (2), a graph $G(\mathbf{s})$ is pairwise-stable if and only if it is pairwise-Nash.

Proof: The proof is very similar to that of Theorem 3.1. We once again only need to verify that a node in a pairwise stable graph does not benefit from deleting multiple links. For a pairwise stable graph $G(\mathrm{~s})$, since there are no unilateral link announcements,

$$
C_{i}^{(2)}(\mathbf{s})=\sum_{j \mid(i, j) \in B(\mathbf{s})} c_{i j}+\sum_{j \mid(i, j) \notin B(\mathbf{s})} \hat{c}_{i j}(\mathbf{s}) .
$$

The marginal cost of node $i$ from deleting link $(i, k)$ is non-negative i.e.,

$$
\begin{array}{r}
C_{i}^{(2)}(\mathbf{s}-(i, k))-C_{i}^{(2)}(\mathbf{s})=-c_{i k}+\hat{c}_{i k}(\mathbf{s}-(i, k)) \\
+\sum_{j \in N_{i k}} \hat{c}_{i j}(\mathbf{s}-(i, k))-\hat{c}_{i j}(\mathbf{s}) \geq 0,
\end{array}
$$

where $N_{i k}=\left\{j \in N \mid(i, j) \notin B(\mathbf{s}), \hat{c}_{i j}(\mathbf{s}-(i, k))>\right.$ $\left.\hat{c}_{i j}(\mathbf{s})\right\}$. Similarly, the marginal cost of deleting link $(i, l)$ is also non-negative and can be obtained by replacing $k$ with $l$ in (9) and $N_{i l}=\left\{j \in N \mid(i, j) \notin B(\mathbf{s}), \hat{c}_{i j}(\mathbf{s}-(i, l))>\right.$ $\left.\hat{c}_{i j}(\mathbf{s})\right\}$. Note that the sets $N_{i k}$ and $N_{i l}$ are disjoint since the least-cost path to any node from node $i$ cannot include two direct links of node $i$. The marginal cost of deleting two links $(i, k)$ and $(i, l)$ can then be expressed as

$$
\begin{aligned}
C_{i}^{(2)}(\mathbf{s}-\{ & (i, k),(i, l)\})-C_{i}^{(2)}(\mathbf{s}) \\
=- & c_{i k}+\hat{c}_{i k}(\mathbf{s}-(i, k))-c_{i l}+\hat{c}_{i l}(\mathbf{s}-(i, k)) \\
& +\sum_{j \in N_{i k}} \hat{c}_{i j}(\mathbf{s}-\{(i, k),(i, l)\})-\hat{c}_{i j}(\mathbf{s}) \\
& +\sum_{j \in N_{i l}} \hat{c}_{i j}(\mathbf{s}-\{(i, k),(i, l)\})-\hat{c}_{i j}(\mathbf{s}) \\
& +\sum_{j \in N_{i k l}} \hat{c}_{i j}(\mathbf{s}-\{(i, k),(i, l)\})-\hat{c}_{i j}(\mathbf{s})
\end{aligned}
$$

where $N_{i k l}=\left\{j \in N \cap\left(N_{i k} \cup N_{i l}\right)^{c} \mid(i, j) \notin\right.$ $\left.B(\mathbf{s}), \hat{c}_{i j}(\mathbf{s}-\{(i, k),(i, l)\})>\hat{c}_{i j}(\mathbf{s})\right\}$. Therefore,

$$
\begin{aligned}
C_{i}^{(2)}(\mathbf{s}-\{( & (i, k),(i, l)\})-C_{i}^{(2)}(\mathbf{s}) \\
\geq- & c_{i k}+\hat{c}_{i k}(\mathbf{s}-(i, k))-c_{i l}+\hat{c}_{i l}(\mathbf{s}-(i, k)) \\
& +\sum_{j \in N_{i k}} \hat{c}_{i j}(\mathbf{s}-\{(i, k),(i, l)\})-\hat{c}_{i j}(\mathbf{s}) \\
& +\sum_{j \in N_{i l}} \hat{c}_{i j}(\mathbf{s}-\{(i, k),(i, l)\})-\hat{c}_{i j}(\mathbf{s})
\end{aligned}
$$

$$
\begin{aligned}
& \geq-c_{i k}+\hat{c}_{i k}(\mathbf{s}-(i, k)) \\
& \quad+\sum_{j \in N_{i k}} \hat{c}_{i j}(\mathbf{s}-(i, k))-\hat{c}_{i j}(\mathbf{s}) \\
& \quad-c_{i l}+\hat{c}_{i l}(\mathbf{s}-(i, k)) \\
& \quad+\sum_{j \in N_{i l}} \hat{c}_{i j}(\mathbf{s}-(i, l))-\hat{c}_{i j}(\mathbf{s}) \\
& =C_{i}^{(2)}(\mathbf{s}-(i, k))-C_{i}^{(2)}(\mathbf{s}) \\
& \quad+C_{i}^{(2)}(\mathbf{s}-(i, l))-C_{i}^{(2)}(\mathbf{s}) \\
& \geq 0 .
\end{aligned}
$$

The above argument easily extends to the case of multiple link deletions and therefore a pairwise stable graph is also pairwise-Nash for the BCG with cost function $C_{i}^{(2)}(\mathbf{s})$.

We now define a specific strategy profile and a corresponding graph that are of interest in the sequel.

Definition 3.7: Let $G\left(\mathrm{~s}^{*}\right)$ be the maximal graph such that each link $(i, j) \in B\left(\mathbf{s}^{*}\right)$ is the least-cost path among all paths between nodes $i$ and $j$ in the complete graph $K_{n}$ and $\mathbf{s}^{*}$ does not contain any unilateral announcements. We call $\mathbf{s}^{*}$ the stable energy-efficient strategy profile and $G\left(\mathbf{s}^{*}\right)$ the stable energy-efficient graph.

Unlike the multitude of trees that are pairwise-Nash graphs for the BCG with cost function $C_{i}^{(1)}(\mathbf{s})$, the stability and efficiency properties of the BCG with cost function $C_{i}^{(2)}(\mathbf{s})$ are different as stated in the following theorem.

Theorem 3.8: For the BCG with cost function $C_{i}^{(2)}(\mathbf{s})$ as in (2), $G\left(\mathbf{s}^{*}\right)$ is the unique pairwise-Nash graph and is also the unique socially efficient graph.

Proof: Using Theorem 3.6, we only need to show that $G\left(\mathbf{s}^{*}\right)$ is the unique pairwise stable graph. From the definition of $G\left(\mathbf{s}^{*}\right)$, a link $(i, j) \in B\left(\mathbf{s}^{*}\right)$ is the least-cost path between nodes $i$ and $j$ in the complete graph over the set $N$. Therefore $c_{i j} \leq \hat{c}_{i j}(\mathbf{s})$ for any strategy profile $\mathbf{s}$ such that $(i, j) \notin B(\mathbf{s})$ and, in particular, $C_{i}^{(2)}\left(\mathbf{s}^{*}-\right.$ $(i, j)) \geq C_{i}^{(2)}\left(\mathbf{s}^{*}\right)$. Therefore every pairwise stable graph must include every link $(i, j) \in B\left(\mathbf{s}^{*}\right)$ i.e., the graph $G\left(\mathbf{s}^{*}\right)$ is a subgraph of every pairwise stable graph.

Adding a link $(i, j) \notin B\left(\mathbf{s}^{*}\right)$, to $G\left(\mathbf{s}^{*}\right)$ will only result in an increase in the cost functions $C_{i}^{(2)}\left(\mathbf{s}^{*}\right)$ and $C_{j}^{(2)}\left(\mathbf{s}^{*}\right)$ by $c_{i j}$ because this link does not decrease the power cost of any of the paths in $G\left(\mathbf{s}^{*}\right)$. The graph $G\left(\mathbf{s}^{*}\right)$ is therefore the unique pairwise stable graph or equivalently the unique pairwise-Nash graph.

From (2), the socially efficient graph does not contain any unilateral link announcements and the social cost of a graph $G(\mathbf{s})$ without such announcements can be written as

$$
\begin{aligned}
C^{(2)}(\mathbf{s})=\sum_{i \in N} C_{i}^{(2)}(\mathbf{s})= & 2 \sum_{(i, j) \in B(\mathbf{s})} c_{i j}(\mathbf{s}) \\
& +2 \sum_{(i, j) \notin B(\mathbf{s})} \hat{c}_{i j}(\mathbf{s}) .
\end{aligned}
$$

Using Definition 3.7, it is easy to see that $C^{(2)}(\mathbf{s})$ above is minimized if and only if $\mathbf{s}=\mathbf{s}^{*}$ i.e., the stable energyefficient graph is the unique socially efficient graph. 


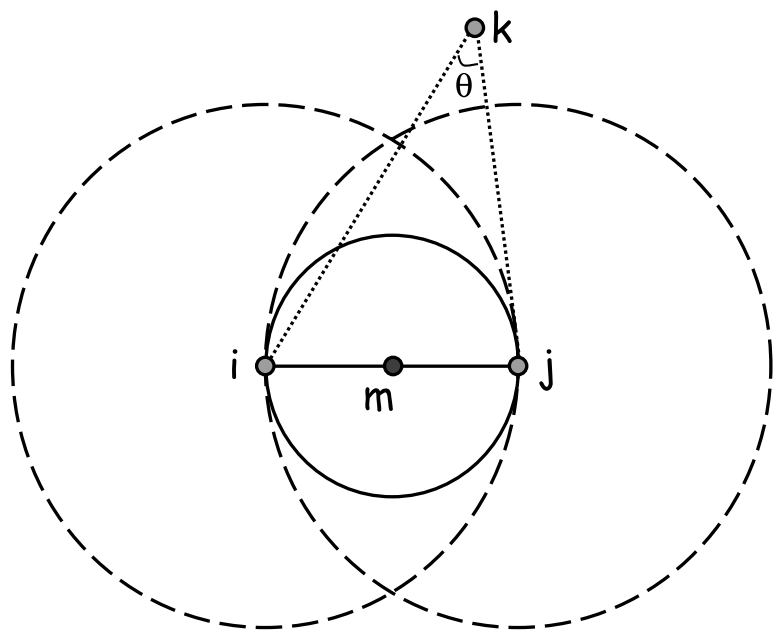

Fig. 2. Condition for Link Announcement

Since the BCG with cost function $C_{i}^{(2)}(\mathbf{s})$ results in a unique stable and energy-efficient network, it is important to see how the cost function can be implemented in practice. We do not delve into these issues in detail in this paper but as mentioned in Section I-C, one implementation approach is to use 'virtual energy currency' similar to the nuglets-based Packet Purse Model of [8]. Further, reputation-systems [15] approaches can be brought to bear to ensure that nodes in the network do not deviate from their pairwise-Nash behavior and not forward data that other nodes route through them. Henceforth, we focus on the stable energy-efficient graph due to its unique stability and efficiency properties. We next describe a simple distributed algorithm for network formation for the free-space path loss model ( $\alpha=2$ in the link power cost).

Algorithm 1: Player $i$ announces $s_{i j}=1$ if and only if no other player $k$ is physically located within a circular region of diameter $d_{i j}$ centered at the mid-point of the line segment joining players $i$ and $j$ in the plane.

The condition for link announcement is illustrated in Fig. 2 where the bold circle represents the circular region of diameter $d_{i j}$, mentioned in the above algorithm, and centered at midpoint $m$ of the line segment joining players $i$ and $j$ in the plane. The following proposition states some of the important properties of the graph formed using this simple distributed algorithm.

Theorem 3.9: Algorithm 1 generates a unique strategy profile and an associated graph that is a supergraph of the stable energy-efficient graph $G\left(\mathbf{s}^{*}\right)$.

Proof: From Definition 3.7, every link in the stable energy-efficient graph $G\left(\mathbf{s}^{*}\right)$ is the least cost path between its end nodes. Using the cosine rule and from Fig. $2, d_{i j}^{2}=$ $d_{i k}^{2}+d_{k j}^{2}-2 d_{i k} d_{k j} \cos \theta \geq d_{i k}^{2}+d_{k j}^{2}$ for $\theta>\pi / 2$. This is true if node $k$ lies within the circle with diameter $d_{i j}$ and centered at the mid-point of the line segment joining players $i$ and $j$. Thus a necessary condition for a link to be the least cost path between its end nodes (and thus be a

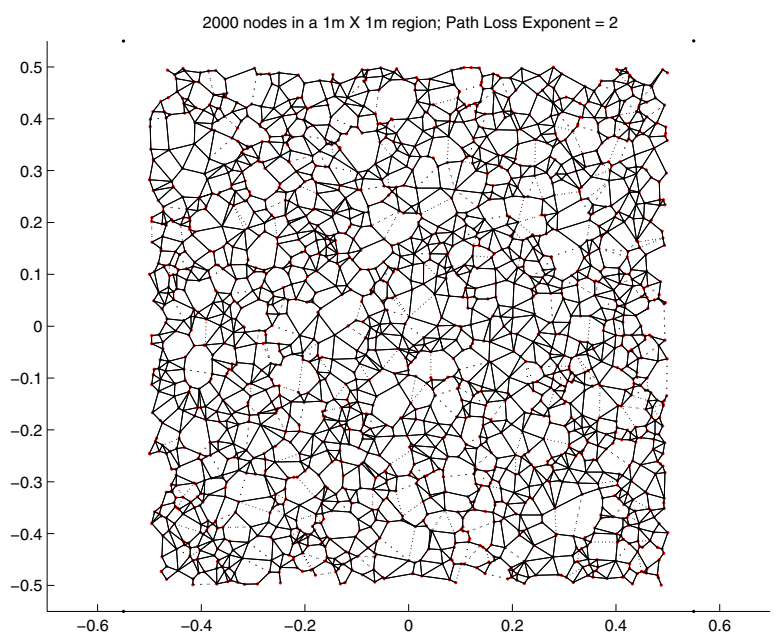

Fig. 3. The stable energy-efficient network $G\left(\mathbf{s}^{*}\right)$ and the network formed by Algorithm 1

part of $G\left(\mathbf{s}^{*}\right)$ ) is that no node be present within this circular region around the link. Algorithm 1 therefore results in a graph that includes every link in the stable energy-efficient graph and more.

We now illustrate the stable energy-efficient graph and the network formed using Algorithm 1. Consider 2000 wireless devices uniformly and randomly deployed in a $1 \mathrm{~m} \times 1 \mathrm{~m}$ region. Fig. 3 shows a network among these nodes with two kinds of edges - bold and dotted. The stable energy-efficient network $G\left(\mathbf{s}^{*}\right)$ among these nodes is the network formed with the bold edges while the network formed using Algorithm 1 includes the dotted edges as well. Numerically, there 3624 links in $G\left(\mathbf{s}^{*}\right)$ while there are an additional 231 links in the network formed by Algorithm 1 i.e., about $6.4 \%$ extra links. It has been observed that around $6 \%$ percent extra links are formed in the network using Algorithm 1 compared to the stable energy-efficient network even for varying network sizes consisting of up to 100000 nodes.

\section{RANDOM POINT-TO-POINT TRAFFIC}

In the bilateral connection game discussed so far, the focus was only on connectivity among the players involved with an infinite cost for not being connected to any node. We showed that the stable energy-efficient graph $G\left(\mathbf{s}^{*}\right)$ is of particular interest since it is a pairwise-Nash graph for both the cost functions that we considered. In the formation of this graph, we were only interested in transmission power costs of each link and did not explicitly consider the effects of interference on simultaneous transmissions. In this section, we study the maximum achievable throughput for each of $n$ random point to point flows in the stable energy-efficient network and comment on how it compares with recent results on capacity of wireless networks with a similar traffic model [9]. In addition, we also discuss the power-law or scale-free behavior for the number of flows going through any node in this network. 
Consider $n$ nodes randomly but uniformly deployed on a unit square and connected as the minimum stable energy graph $G\left(\mathbf{s}^{*}\right)$. Each node in the network wishes to communicate with a destination node, which is chosen to be the node closest to a uniformly random point in the region. There are thus $n$ point to point flows in the network and packets are assumed to be routed along the path with the least power cost. We wish to compute the maximum common throughput of each flow that is achievable in this network.

Theorem 4.1: The longest link in the stable energyefficient network $G\left(\mathbf{s}^{*}\right)$ is $O(\sqrt{\log n / n})$ units long almost surely.

Proof: As noted in the proof of Theorem 3.9, if a node $k$ lies within the circular region of diameter $d_{i j}$ and centered at the midpoint $m$ of nodes $i$ and $j$ as shown in Fig. 2, $\left(d_{i k}^{2}+d_{k j}^{2}\right)^{1 / 2} \leq d_{i j}$. Since $\left(d_{i k}^{\alpha}+d_{k j}^{\alpha}\right)^{1 / \alpha} \leq$ $\left(d_{i k}^{2}+d_{k j}^{2}\right)^{1 / 2}$ for $\alpha \geq 2$, a necessary condition for a link $d_{i j}$ to be a part of the stable energy-efficient network is that no node lie within this circular region.

No link can be longer than $\sqrt{8 A_{n}}$ since the circular region around such a link will necessarily include at least one cell, and from Lemma 3.4 every cell is non-empty almost surely for $A_{n}=\frac{a \log n}{n}, a>2$.

For the random point to point traffic model comprising of a randomly chosen destination for each source node, the following lemma characterizes the number of sourcedestination lines (i.e., straight lines each joining a source to its randomly chosen destination) through any cell.

Lemma 4.2: (Lemma 3 of [11]) The number of sourcedestination lines passing through any cell is $O(\sqrt{n \log n})$, almost surely.

Theorem 4.3: Node degree in the stable energy-efficient network $G\left(\mathbf{s}^{*}\right)$ is $O(\log n)$ almost surely.

Proof: From Theorem 4.1, the maximum link length in $G\left(\mathbf{s}^{*}\right)$ is $O(\sqrt{\log n / n})$. Specifically, a circle with the maximum link length as diameter encloses at most 4 cells. Since each cell has $O(\log n)$ nodes almost surely from Lemma 3.4, so does a circular region around a node with maximum link length as the radius. Therefore each node connects to $O(\log n)$ nodes almost surely.

Using the protocol model of [9], communication between two nodes is successful if there are no other transmitters within a distance $(1+\Delta) l_{\max }$ of the receiver, where $\Delta>0$ represents a guard zone specified by the protocol and $l_{\max }$ is the longest communication link in the network. For the stable energy-efficient network $G\left(\mathbf{s}^{*}\right), l_{\max }$ is $O(\sqrt{\log n / n})$ from Theorem 4.1 and there are at most $O(\log n)$ other nodes within a distance $(1+\Delta) l_{\max }$ of a receiver that cannot transmit at the same time. Using Lemma 4.2, the maximum number of source-destination lines passing through the $O(\log n)$ interferers around any receiver is $O(\sqrt{n \log n})$. Based on Monte-Carlo simulations we observe that, as the network size increases, the leastcost path between every random source-destination pair approaches the straight line path joining them. We therefore

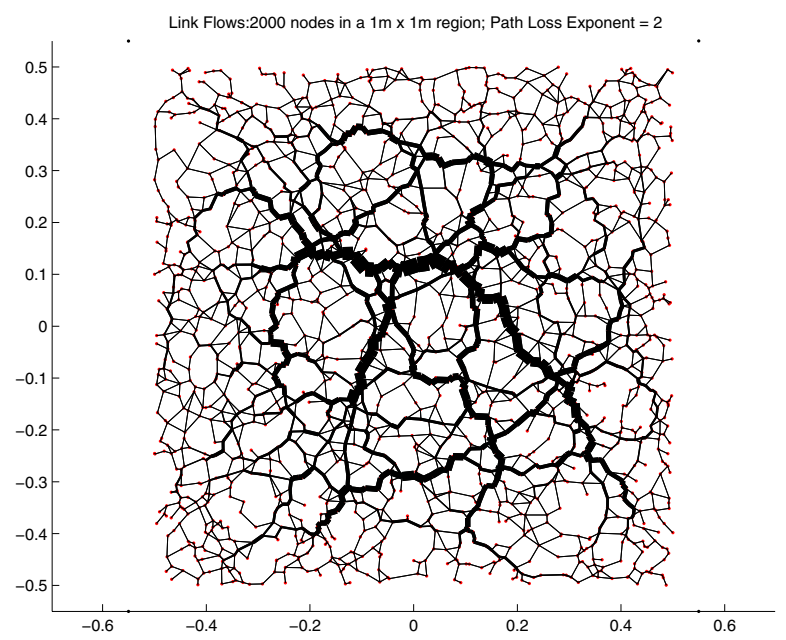

Fig. 4. Point to point flows in the stable energy-efficient graph $G\left(\mathbf{s}^{*}\right)$ (thicker edge indicates more flows through that link).

conjecture that asymptotically the number of least-cost paths through the $O(\log n)$ interferers around any receiver also $O(\sqrt{n \log n})$. Using a slotted-time schedule with each period consisting of $O(\sqrt{n \log n})$ slots, each flow can be scheduled for one slot in every time period and therefore we conjecture that a per-flow throughput $\Omega(1 / \sqrt{n \log n})$ is achievable in the stable energy-efficient network $G\left(\mathbf{s}^{*}\right)$.

For the stable energy-efficient network connecting 2000 nodes as shown in Fig. 3, we consider a flow from each node to a random destination. Fig. 4 illustrates the routing of these random point to point flows through this network with the thickness of each link being proportional to the number of flows routed through that link. Due to the deployment in a bounded region, nodes closer to the center of the square region route more flows than those farther away from the center. For this network, using maximum likelihood estimation based curve fitting [16], we observe a power-law for the number of flows routed by each node, as shown in the log-log plot of Fig. 5. We observe such a power-law consistently even for larger networks as shown in Fig. 6 for a 50000-node network, where the exponent is equal to 1.26. Such a power-law characteristic, also termed as "scale-free" behavior, has been recently observed for the node degree of large networks like the Internet and other social networks. The practical implications of the powerlaw behavior for the stable energy-efficient network is that while most nodes route only a few flows each, there is a significant fraction of nodes that each route a large number of flows as seen in the 'long tail' of the power-law fit.

\section{CONCLUSiOn AND Future WORK}

In this work, we studied the possibility of a bunch of selfish energy-constrained wireless devices forming a communication network among themselves so that any two nodes are connected to each other either directly or through other devices. We used a non-cooperative bilateral connection game framework to model network formation 


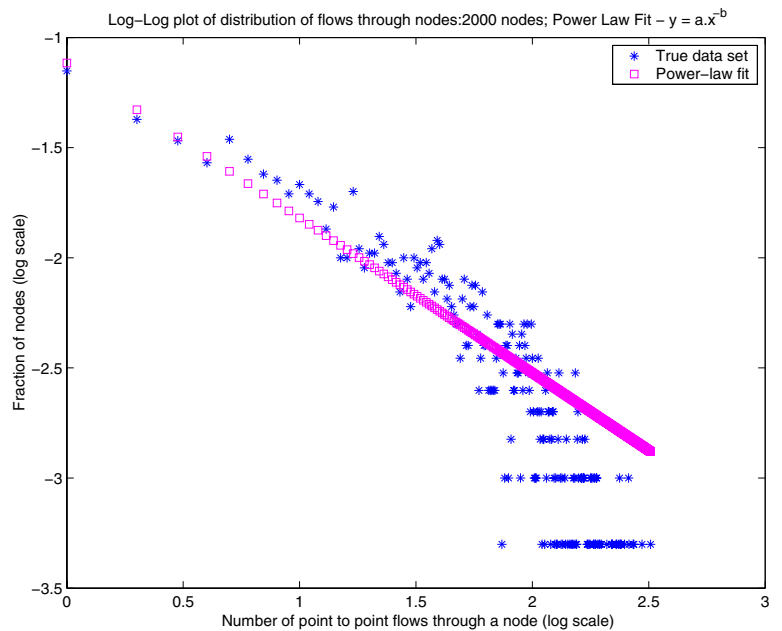

Fig. 5. Distribution of flows and power-law fit in $G\left(\mathbf{s}^{*}\right)$ consisting of 2000 nodes

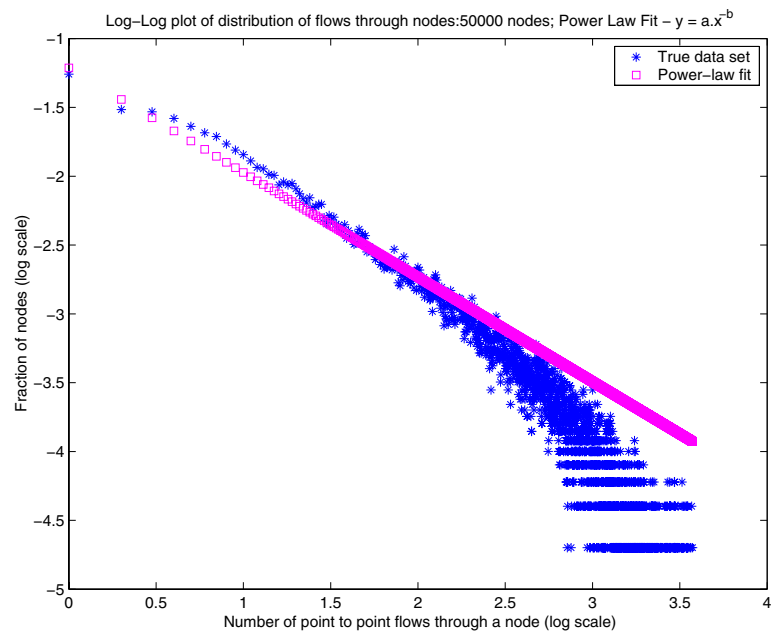

Fig. 6. Distribution of flows and power-law fit in $G\left(\mathbf{s}^{*}\right)$ consisting of 50000 nodes

in which links are formed between nodes based on mutual consent. For a BCG in which devices choose their individual strategies to remain connected by minimizing only their direct transmission power costs, we showed that the price-of-anarchy is unbounded in the network size. We then proposed a BCG with an alternate cost structure in which each device additionally pays the transmission power costs incurred by other devices for its own traffic. We showed that a unique network structure emerges in this game that is stable as well as socially efficient. We then studied the achievable throughput for random point-to-point traffic in this stable energy-efficient network. When the nodes of a network are located in a bounded planar region, the distribution of point-to-point flows through the nodes exhibits a scale-free behavior.

For random point-to-point traffic over the stable energyefficient network, our conjecture that the achievable throughput is the same (in the order sense) as the capacity of such wireless networks needs to be proven as a part of future work. In particular, it remains to be shown analytically that the total distance traversed by a least cost path in the stable energy-efficient network is asymptotically close to the euclidean distance between the source and destination of the path. The second cost function $C_{2}(\mathbf{s})$ proposed in our model implicitly assumes a uniformly random distribution of flows from each node to any other node. Another direction of future work is to study BCGs with cost functions that allow non-uniform distribution of flows between pairs of nodes or alternately link costs that depend on the amount of traffic through them. An instance of a non-uniform distribution could be that a node only wishes to be connected to a subset of nodes [17] and not all the nodes.

\section{ACKNOWLEDGMENT}

The authors would like to acknowledge the support of NSF grants CCF-0634973 and CCF-0721826.

\section{REFERENCES}

[1] A. Fabrikant, A. Luthra, E. Maneva, C. H. Papadimitriou, and S. Shenker, "On a Network Creation Game," in PODC, 2003.

[2] E. Koutsoupias and C. H. Papadimitriou, "Worst-case equilibria," Lecture Notes in Computer Science, vol. 1563, pp. 404-413, 1999.

[3] R. Myerson, Game Theory: Analysis of Conflict. Cambridge, MA: Harvard University Press, 1991.

[4] J. Corbo and D. Parkes, "The Price of Selfish Behavior in Bilateral Network Formation," in PODC, July 2005.

[5] A. Calvó-Armengol and R. Ilkiliç, "Pairwise-Stability and Nash Equilibria in Network Formation Games," Mar 2006, Working Paper Series.

[6] M. O. Jackson, "A survey of models of network formation: stability and efficiency," in Group Formation in Economics; Networks, Clubs and Coalitions, G. Demange and M. Wooders, Eds. Cambridge University Press, 2004, ch. 1.

[7] S. Mathur, L. Sankaranarayanan, and N. Mandayam, "Coalitions in cooperative wireless networks," Submitted to IEEE Jrnl. on Selected Areas in Comm.

[8] L. Blazevic, L. Buttyan, S. Capkun, S. Giordano, J.-P. Hubaux, and J. Y. L. Boudec, "Self organization in mobile ad hoc networks: the approach of terminodes," IEEE Commun. Mag., vol. 39, no. 6, pp. 166-174, June 2001.

[9] P. Gupta and P. R. Kumar, "Capacity of wireless networks," IEEE Trans. Inform. Theory, vol. 46, no. 2, pp. 388-401, Mar 2000.

[10] S. Kulkarni and P. Viswanath, "A deterministic approach to throughput scaling in wireless networks," IEEE Trans. Inform. Theory, vol. 50, no. 6, pp. 1041-1049, June 2004.

[11] A. E. Gamal, J. Mammen, B. Prabhakar, and D. Shah, "Throughputdelay trade-off in wireless networks," in Proc. IEEE Infocom, 2004.

[12] D. Watts and S. Strogatz, "Collective dynamics of 'small world' networks," Nature, vol. 393, pp. 440-442, 1998.

[13] A. Barabási and R. Albert, "Emergence of scaling in random networks," Science, vol. 286, pp. 509-512, 1999.

[14] M. O. Jackson and B. W. Rogers, "The Strategic Formation of Large Networks: When and Why do We See Power Laws and Small Worlds," 2004, preprint: Caltech. [Online]. Available: http://www.hss.caltech.edu/ jacksonm/netpower.pdf

[15] P. Resnick, R. Zeckhauser, E. Friedman, and K. Kuwabara, "Reputation systems," Communications of the ACM, vol. 43, no. 12, p. 4548, 2000.

[16] M. L. Goldstein, S. A. Morris, and G. G. Yen, "Problems with fitting to the power-law distribution," The European Physical Journal B, vol. 41, p. 255258, 2004.

[17] S. Eidenbenz, V. S. A. Kumar, and S. Zust, "Equilibria in topology control games for ad hoc networks," in DIALM-POMC '03. Proceedings of the 2003 joint workshop on Foundations of Mobile Computing. 